\begin{tabular}{|c|c|}
\hline Title & Entanglement generation by communication using squeezed states \\
\hline Author(s) & Matsuoka, Fumiaki; Tomita, A kihisa; Okamoto, A tsushi \\
\hline Citation & $\begin{array}{l}\text { Physical Review A, 88(2), } 022313 \\
\text { https://doi.org/10.1103/PhysRevA .88.022313 }\end{array}$ \\
\hline Issue Date & $2013-08-12$ \\
\hline Doc URL & http:/hdl.handle.net/2115/53294 \\
\hline Rights & @2013 A merican Physical Society \\
\hline Type & article \\
\hline File Information & Phy sRevA . 88.022313.pdf \\
\hline
\end{tabular}

Instructions for use 


\title{
Entanglement generation by communication using squeezed states
}

\author{
Fumiaki Matsuoka, ${ }^{*}$ Akihisa Tomita, ${ }^{\dagger}$ and Atsushi Okamoto \\ Graduate School of Information Science and Technology, Hokkaido University, Kital4-Nishi9, Kita-ku, Sapporo 060-0814, Japan
}

(Received 23 March 2013; published 12 August 2013)

\begin{abstract}
In order to improve the error probability of generating entanglement by communication for quantum computation, we propose the use of squeezed light. When generating entanglement between two atoms by communication, the error probability can be reduced by increasing the distance between quantum states of probe light in phase space. The phase rotation of light depends on the atom-photon coupling strength and the light amplitude, which are limited in practice. A large error probability has been expected for coherent probe light. If we assume typical values of light amplitude and phase rotation, $\alpha=100$ and $\theta=0.01$, the error probability is estimated to be $P_{\mathrm{coh}}^{(\mathrm{min})}=0.14$ and $P_{\mathrm{coh}}^{(\mathrm{hom})}=0.23$ for minimum error discrimination and homodyne measurements. The error probability can be reduced to $P_{\text {squ }}^{(\min )}=1.73 \times 10^{-8}$ and $P_{\text {squ }}^{(\text {hom })}=4.09 \times 10^{-5}$ using squeezed coherent light, where the same values of the mean photon number and the phase rotation angle are assumed for the coherent light probe. These values satisfy the requirements for scalable quantum computation.
\end{abstract}

DOI: 10.1103/PhysRevA.88.022313 PACS number(s): 03.67.Bg, 03.65.Ud, 03.67.Lx, 03.67.Hk

\section{INTRODUCTION}

Quantum computers can achieve a drastic reduction of computational complexity compared to classical computers [1]. Currently, a small-scale quantum circuit with several quantum bits (qubits) has been achieved [2]. However, construction of a practical quantum computer based on the quantum circuit model is still difficult to implement. For example, one obstacle to the implementation of solid-state qubit systems with nearest-neighbor interaction is the requirement of a large number of swap operations, necessary for interactions between remote qubits. In recent years, measurement-based quantum information processing has been proposed to overcome this problem in order to implement complicated quantum circuits. In the measurement-based model, quantum computation is realized by the measurement of a quantum bit and a onequbit unitary transformation according to the measurement outcomes [3]. Since complicated control techniques become unnecessary, the measurement-based model is expected to simplify the structure of a quantum computer. The remaining problem is the preparation of multiqubit entangled states prior to quantum computation.

Entanglement generation by communication [4] has been proposed as an efficient entangler that can be used for measurement-based quantum information processing. An electromagnetic field (i.e., a quantum bus or qubus) interacts with quantum memories, and the phase rotation of the field is measured after the interaction. Entanglement is generated between quantum memories by postselection according to the measurement outcome. The qubus acts as a probe for the memory states. The qubus interaction, unlike neighbor interaction, can generate entanglement between remote quantum memories. Since errors in entanglement generation occur due to overlaps of qubus states, the error probability can be improved by increasing the distance between the quantum states of light in phase space. When three-level atoms are used

\footnotetext{
*matsuoka@optnet.ist.hokudai.ac.jp

†tomita@ist.hokudai.ac.jp
}

for the quantum memories, the phase rotation of light depends on the atom-light coupling strength and the light amplitude. However, the light amplitude and the phase rotation angle are limited in practice since a weak coupling [5] and a small absorption rate are required [6]. On the other hand, coherent light, which has been considered for the qubus, appears to have difficulties in satisfying the requirements of low error probability for large-scale quantum computation.

In this report, we propose the use of squeezed coherent light to reduce the overlap and improve the error probability of entanglement generation for quantum computation. Squeezed light has been studied for qubuses and a related system in the context of quantum repeaters $[7,8]$. The use of squeezed light and the displacement operation is predicted to improve the fidelity and success probability of dispersive interaction to 0.89 and $40 \%$, respectively, for a node interval of $10 \mathrm{~km}$, in comparison to values of 0.77 and $36 \%$ for coherent light [7]. In quantum repeaters, however, photon loss in long-distance transmission affects the performance, limiting the advantages of squeezed light. For quantum computing, as we propose, since the distance between quantum memories is small and the transmission loss can be negligible, squeezed light will work more effectively. Moreover, Praxmeyer and van Loock [8] employed homodyne measurements and window functions for quantum state discrimination to obtain the final state with a high fidelity to the ideal Bell state, which reduced the success probability to less than $1 / 2$. It is not clear, however, whether quantum error correction, such as Knill's error correcting $C_{4} / C_{6}$ architecture $[9,10]$, can be implemented with a qubus for a success probability below $1 / 2$.

Therefore, minimum error discrimination, which maximizes the success probability without restricting the discernment error, is more suitable for quantum computation. It is well known that the Helstrom bound provides the error probability for minimum error discrimination for two nonorthogonal states. However, entanglement generation by the qubus requires discrimination between three states. In the following research, we developed a theory on three-state minimum error discrimination and calculated the error probability for entanglement generation with squeezed light for 
quantum computation. We also calculated the error probability with homodyne measurements. It is shown that the error probability can be reduced to satisfy the requirements for quantum computing in both discrimination methods.

The report is structured as follows. We will review the atom-photon interaction briefly in Sec. II. After this, we will obtain the optimal positive operator value measurement (POVM) for the discrimination of three pure states and will show the improvement of the error probability of entanglement generation with squeezed states in Sec. III. In Sec. IV, we will yield a homodyne measurement scheme for three-state discrimination and calculate the error probability. Finally, we will examine the obtained values for reliable quantum computation and conclude the report in Sec. V.

\section{ATOM-PHOTON INTERACTION}

In entanglement generation by communication, the phase rotation of the probe light according to the atomic states should be measured. In the following section, we will briefly review a model of interaction between a photon and a three-level atom used to calculate the phase rotation $[4,5]$. We assume that the atom has the lower energy levels $|0\rangle$ and $|1\rangle$ and an excited state $|e\rangle$, where only the transition $|1\rangle \longleftrightarrow|e\rangle$ is allowed $[11,12]$. The effective Hamiltonian of this atom-photon system can be reduced to

$$
\hat{H}=\hbar \frac{\omega_{0}}{2} \hat{\sigma}_{z}+\hbar \omega_{c} \hat{a}^{\dagger} \hat{a}+\frac{\hbar \Omega}{2}\left(\hat{a} \hat{\sigma}_{+}+\hat{a}^{\dagger} \hat{\sigma}_{-}\right)
$$

by adiabatic elimination. Here, $\hat{\sigma}_{z}=|1\rangle\langle 1|-| 0\rangle\langle 0|, \hat{\sigma}_{+}=$ $|1\rangle\left\langle 0\left|, \hat{\sigma}_{-}=\right| 0\right\rangle\langle 1|, \hbar \omega_{0}$ is the energy difference between the ground state and the excited state, $\omega_{c}$ is the frequency of the quantized cavity mode, and $\Omega$ is the Rabi frequency. We can then move to the interaction picture using the unitary transformation $\hat{U}_{0}(t)=\exp \left[-i \frac{\omega_{0}}{2} \sigma_{z} t-i \omega_{c} \hat{a}^{\dagger} \hat{a} t\right]$. The state in the interaction picture satisfies the following equation of motion:

$$
\frac{d|\psi(t)\rangle_{I}}{d t}=-\frac{i}{\hbar} \hat{H}_{I}(t)|\psi(t)\rangle_{I}
$$

where

$$
\hat{H}_{I}(t)=\frac{\hbar \Omega}{2}\left(\hat{a} \hat{\sigma}_{+} e^{-i \Delta t}+\hat{a}^{\dagger} \hat{\sigma}_{-} e^{i \Delta t}\right)
$$

and $\Delta=\omega_{c}-\omega_{0}$. The formal solution of Eq. (2) up to the second order is given by

$$
\begin{aligned}
|\psi(t)\rangle_{I}= & {\left[1-\frac{i}{\hbar} \int_{0}^{t} d t_{1} \hat{H}_{I}\left(t_{1}\right)+\frac{1}{2}\left(-\frac{i}{\hbar}\right)^{2} \int_{0}^{t} d t_{2}\right.} \\
& \left.\times \int_{0}^{t_{2}} d t_{1} \hat{H}_{I}\left(t_{2}\right) \hat{H}_{I}\left(t_{1}\right)\right]|\psi(0)\rangle_{I} .
\end{aligned}
$$

We assume a large detuning and a not too large mean photon number $\bar{n}$ in the cavity mode, i.e., $\Delta t \gg 1$ and $\sqrt{\bar{n}} / \Delta=$ $\Omega / \Delta \ll 1$. This is called the dispersive limit of interaction. In this limit, the second term in Eq. (4) is proportional to $\Omega / \Delta$ and can be neglected. Furthermore, the third term is given by

$$
\int_{0}^{t} d t_{2} \int_{0}^{t_{2}} d t_{1} \hat{H}_{I}\left(t_{2}\right) \hat{H}_{I}\left(t_{1}\right) \approx-i \frac{\hbar^{2} \Omega^{2}}{4 \Delta}\left(\hat{\sigma}_{+} \hat{\sigma}_{-}+\hat{a}^{\dagger} \hat{a} \hat{\sigma}_{z}\right) t
$$

Eventually, Eq. (4) can be written as

$$
\begin{aligned}
|\psi(t)\rangle_{I} & =\left[1-\frac{i}{\hbar} \hat{H}_{\text {disp }} t\right]|\psi(0)\rangle_{I}, \\
\hat{H}_{\text {disp }} & =\hbar \chi\left(\hat{\sigma}_{+} \hat{\sigma}_{-}+\hat{a}^{\dagger} \hat{a} \hat{\sigma}_{z}\right),
\end{aligned}
$$

where $\chi=g^{2} / \Delta$ for a coupling constant $g=$ $-i d \sqrt{\hbar \omega_{c} / 2 \epsilon_{0} V} / \hbar$ with a dipole moment $d$ between the excited state and the ground state, the volume of the mode is $V$, and the dielectric constant is $\epsilon_{0}$. The interaction Hamiltonian $\hat{H}_{\text {disp }}$ is given in the dispersive limit. To go further, since the mean photon number $\bar{n}=\hat{a}^{\dagger} \hat{a} \gg 1$, we can neglect the operator $\hat{\sigma}_{+} \hat{\sigma}_{-}$. Finally, we obtain a unitary operator that conditionally rotates the phase of the photon as

$$
\hat{U}=\hat{I}|0\rangle\langle 0|+\hat{R}(\theta)| 1\rangle\langle 1|,
$$

where $\theta=\chi t$ and $\hat{R}(\theta)=e^{i \theta \hat{n}}$.

\section{IMPROVEMENT OF ERROR PROBABILITY FOR OPTIMAL MEASUREMENT BY THE USE OF SQUEEZED LIGHT}

The process for a qubus with a probe light sequentially interacting with two atoms is shown in Fig. 1(a), and the energy-level scheme for a three-level atom is shown in Fig. 1(b). At $\tau^{\prime \prime}$ in Fig. 1(a), that is, after interacting with the two atoms, the probe light is in a superposition of three states with different phases, as $\left|\psi^{\text {fin }}\right\rangle=\frac{1}{2}|0\rangle|0\rangle|0\rangle_{\mathrm{ph}}+$ $\frac{1}{2}(|0\rangle|1\rangle+|1\rangle|0\rangle) \hat{R}(\theta)|0\rangle_{\mathrm{ph}}+\frac{1}{2}|1\rangle|1\rangle \hat{R}^{2}(\theta)|0\rangle_{\mathrm{ph}}$, where (a)

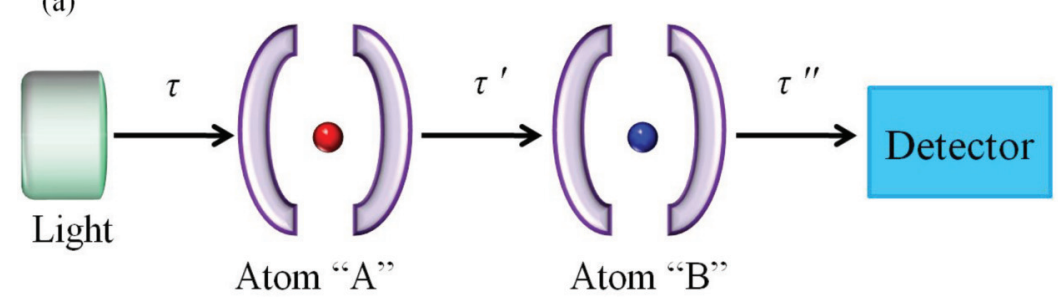

(b)

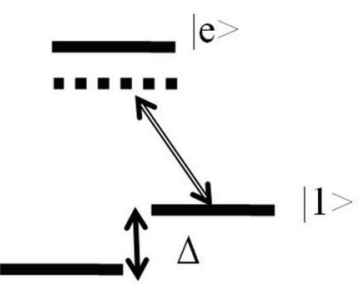

FIG. 1. (Color online) (a) The process of entanglement generation between quantum memories by communication. Light interacts with two atoms, and the phase rotation is measured after the interaction. (b) The energy-level scheme of a $\Lambda$-configuration three-level atom. The phase of light rotates only when the atom is in state $|1\rangle$. 


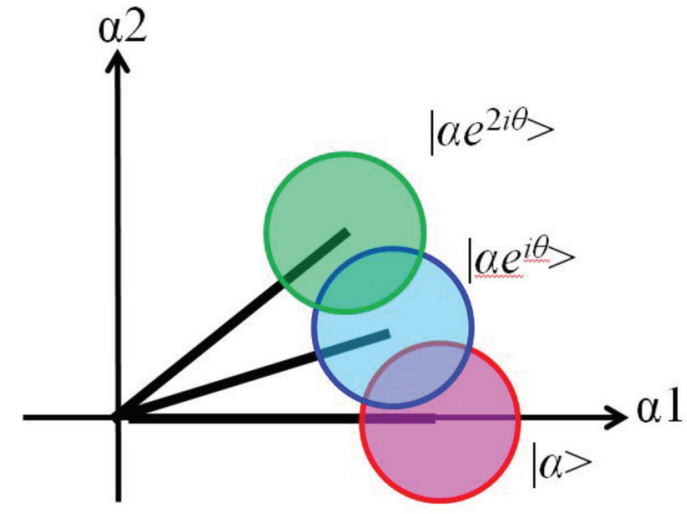

FIG. 2. (Color online) The coherent states in phase space after interacting with atoms $A$ and $B$ [at time $\tau^{\prime \prime}$ in Fig. 1(a)], described using circles. The final state of the total system is given by $\frac{1}{2}|0\rangle|0\rangle|\alpha\rangle+\frac{1}{\sqrt{2}}(|0\rangle|1\rangle+|1\rangle|0\rangle)\left|\alpha e^{i \theta}\right\rangle+\frac{1}{2}|1\rangle|1\rangle\left|\alpha e^{i 2 \theta}\right\rangle$. The atomic entanglement state $|0\rangle|1\rangle+|1\rangle|0\rangle$ is formed by postselecting the rotated coherent state $\left|\alpha e^{i \theta}\right\rangle$. The error in the entanglement generation originates from the overlaps of the circles (i.e., overlaps between the light states).

$|0\rangle_{\mathrm{ph}}$ stands for the initial state of the light. After measuring the light state, the atomic entanglement state is obtained by postselecting the $\hat{R}(\theta)|0\rangle_{\mathrm{ph}}$ outcome. In entanglement generation by communication, a discrimination of quantum states is important, since the error occurs due to overlaps between quantum states of light. To provide a concrete description, we will consider the entanglement generation with a coherent light qubus state. The interaction between the light and atom $A$ creates an entanglement between the atomic states and the light states, as $\frac{1}{\sqrt{2}}|0\rangle|\alpha\rangle+\frac{1}{\sqrt{2}}|1\rangle\left|\alpha e^{i \theta}\right\rangle$ from the initial product state $\frac{1}{\sqrt{2}}(|0\rangle+|1\rangle) \otimes|\alpha\rangle$. Then, the interaction between the light and atom $B$ yields the final state $\left|\psi_{\mathrm{coh}}^{\mathrm{fin}}\right\rangle=\frac{1}{2}|0\rangle|0\rangle|\alpha\rangle+$ $\frac{1}{2}(|0\rangle|1\rangle+|1\rangle|0\rangle)\left|\alpha e^{i \theta}\right\rangle+\frac{1}{2}|1\rangle|1\rangle\left|\alpha e^{i 2 \theta}\right\rangle$. The entangled state of the atoms $|0\rangle|1\rangle+|1\rangle|0\rangle$ is formed by postselecting the rotated coherent state $\left|\alpha e^{i \theta}\right\rangle$. The error in the entanglement generation occurs through the error in the light state measurement, i.e., by overlapping between $|\alpha\rangle,\left|\alpha e^{i \theta}\right\rangle$, and $\left|\alpha e^{i 2 \theta}\right\rangle$, as shown in Fig. 2.

For minimum error discrimination of two pure states, the Helstrom bound provides the optimal error probability [13]. However, the Helstrom bound cannot apply for three-state discrimination. Therefore, we now consider the minimum error discrimination for three pure states. The state can be written as

$$
\begin{aligned}
\left|\psi^{\mathrm{fin}}\right\rangle= & \frac{1}{2}|0\rangle_{\mathrm{ph}}|00\rangle_{\mathrm{atom}}+\frac{1}{2}|1\rangle_{\mathrm{ph}}\left(|01\rangle_{\mathrm{atom}}+|10\rangle_{\text {atom }}\right) \\
& +\frac{1}{2}|2\rangle_{\mathrm{ph}}|11\rangle_{\text {atom }},
\end{aligned}
$$

where $|k\rangle_{\text {ph }}(k=0,1,2)$ are probe light states to be measured. We will introduce a POVM for $|k\rangle_{\mathrm{ph}}$ satisfying

$$
\begin{gathered}
\hat{M}_{0}+\hat{M}_{1}+\hat{M}_{2}=\hat{I}, \\
\hat{M}_{0}, \hat{M}_{1}, \hat{M}_{2} \geqslant 0 .
\end{gathered}
$$

The error probability can be described by the events that atomic states are $|01\rangle_{\text {atom }}+|10\rangle_{\text {atom }}$ when the outcome of the measurement is $|0\rangle_{\mathrm{ph}}$ or $|2\rangle_{\mathrm{ph}}$ and that atomic states are $|00\rangle_{\text {atom }}$ or $|11\rangle_{\text {atom }}$ when the outcome of the measurement is $|1\rangle_{\mathrm{ph}}$ :

$$
\begin{aligned}
P_{E}= & \frac{1}{\sqrt{2}} \mathrm{ph}\left\langle 1\left|\left(\frac{\text { atom }\langle 01|+{ }_{\text {atom }}\langle 10|}{\sqrt{2}}\right)\left(\hat{M}_{0}+\hat{M}_{2}\right)\right| \psi^{\text {fin }}\right\rangle \\
& +\frac{1}{2}\left({ } _ { \mathrm { ph } } \left\langle\left.0\right|_{\text {atom }}\left\langle 00\left|\hat{M}_{1}\right| \psi^{\mathrm{fin}}\right\rangle+_{\mathrm{ph}}\left\langle\left. 2\right|_{\text {atom }}\left\langle 11\left|\hat{M}_{1}\right| \psi^{\mathrm{fin}}\right\rangle\right)\right.\right. \\
= & \frac{1}{2}-\operatorname{tr}\left[\hat { M } _ { 1 } \left(\frac{1}{2}|1\rangle_{\mathrm{phph}}\left\langle 1\left|-\frac{1}{4}\right| 0\right\rangle_{\mathrm{phph}}\langle 0|\right.\right. \\
& \left.\left.-\frac{1}{4}|2\rangle_{\mathrm{phph}}\langle 2|\right)\right] \\
= & \frac{1}{2}-\operatorname{tr}\left[\hat{M}_{1} \hat{\Gamma}\right] .
\end{aligned}
$$

The optimal measurement and the minimum error probability can be obtained from the positive maximum eigenvalue and eigenvector of the operator $\hat{\Gamma}$. We can obtain the maximum eigenvalue and eigenvector numerically by solving an eigenvalue equation, $\operatorname{det}|\hat{\Gamma}-\lambda I|$, where the operator $\hat{\Gamma}$ is represented by a matrix. To this end, we will calculate the orthonormal bases $\left\{\left|\phi_{0}\right\rangle,\left|\phi_{1}\right\rangle,\left|\phi_{2}\right\rangle\right\}$ from the linearly independent state vectors $|k\rangle_{\mathrm{ph}}(k=0,1,2)$ using the GramSchmidt method. The state vectors are related to the orthonormal bases as follows:

$$
\begin{gathered}
|0\rangle_{\mathrm{ph}}=\left|\phi_{0}\right\rangle, \\
|1\rangle_{\mathrm{ph}}=a_{0}\left|\phi_{0}\right\rangle+a_{1}\left|\phi_{1}\right\rangle, \\
|2\rangle_{\mathrm{ph}}=b_{0}\left|\phi_{0}\right\rangle+b_{1}\left|\phi_{1}\right\rangle+b_{2}\left|\phi_{2}\right\rangle,
\end{gathered}
$$

where $\left|a_{0}\right|^{2}+\left|a_{1}\right|^{2}=1, \quad\left|b_{0}\right|^{2}+\left|b_{1}\right|^{2}+\left|b_{2}\right|^{2}=1, \quad a_{0}=$ ${ }_{\mathrm{ph}}\langle 0 \mid 1\rangle_{\mathrm{ph}}, a_{1}=\sqrt{1-\left|a_{0}^{*}\right|^{2}}, b_{0}={ }_{\mathrm{ph}}\langle 0 \mid 2\rangle_{\mathrm{ph}}, b_{1}={ }_{\mathrm{ph}}\langle 1 \mid 2\rangle_{\mathrm{ph}}$, and $b_{2}=\sqrt{1-\left|b_{0}\right|^{2}+\left|b_{1}\right|^{2}-\frac{2\left|b_{1}\right|^{2}-a_{0} b_{1} b_{0}^{*}-a_{0}^{*} b_{1}^{*} b_{0}}{\sqrt{1-\left|a_{0}\right|^{2}}}}$. Therefore, the matrix representation in the orthonormal basis of the operator $\hat{\Gamma}$ is

$$
\hat{\Gamma}=\left(\begin{array}{ccc}
-\frac{1}{4}+\frac{\left|a_{0}\right|^{2}}{2}-\frac{\left|b_{0}\right|^{2}}{4} & \frac{a_{0} a_{1}^{*}}{2}-\frac{b_{0} b_{1}^{*}}{4} & -\frac{b_{0} b_{2}^{*}}{4} \\
\frac{a_{1} a_{0}^{*}}{2}-\frac{b_{1} b_{0}^{*}}{4} & \frac{\left|a_{1}\right|^{2}}{2}-\frac{\left|b_{0}\right|^{2}}{4} & -\frac{b_{1} b_{2}^{*}}{4} \\
-\frac{b_{2} b_{0}^{*}}{4} & -\frac{b_{2} b_{1}^{*}}{4} & -\frac{\left|b_{2}\right|^{2}}{4}
\end{array}\right) .
$$

Note that the coefficients in Eq. (15) are given by the inner products, or the overlaps, of the state vectors. Since the fidelity of the two states refers to the inner product of the states, the minimum error probability is estimated from the fidelities of the state. In fact, if these fidelities are zero, then the three states are mutually orthogonal and thus completely discriminable $\left(P_{E}=0\right)$.

The fidelities of the states are given by $F_{\text {coh }}^{\prime}=$ $\left|\left\langle\alpha \mid \alpha e^{i \theta}\right\rangle\right|=\exp \left[-|\alpha|^{2}(1-\cos \theta)\right]\left(=\left|\left\langle\alpha e^{i \theta} \mid \alpha e^{i 2 \theta}\right\rangle\right|\right) \quad$ and $F_{\text {coh }}^{\prime \prime}=\left|\left\langle\alpha \mid \alpha e^{i 2 \theta}\right\rangle\right|$ for coherent states. To improve the error probability, it is necessary to increase the phase rotation angle $\theta$ close to $\pi$, or to strengthen the light amplitude $\alpha$. However, the light amplitude $\alpha$ and the phase rotation angle $\theta$ are limited in practice. This is because it is necessary to consider the dispersive limit condition in order to realize the rotation gate shown in Fig. 1; i.e., a weak coupling $\left(\theta=g^{2} t / \Delta \ll 1\right)$ [5] and a small absorption rate $\left(\alpha^{2} g^{2} / \Delta^{2} \sim 0.01\right)$ [6] are required. In the following, we will consider a diamond nitrogen-vacancy 
center embedded in a photonic crystal [6] to provide a numerical illustration. The coupling constant $g \sim 10^{4}$ (in $\mathrm{MHz}$ ) has been reported [6], and the interaction time $t$ is on the order of nanoseconds in quantum computation [14]. Therefore, the detuning should be set to $\Delta=10$ (in $\mathrm{THz}$ ) to satisfy the weak-coupling condition, and the mean photon number should be $10^{4}(\alpha=100)$ to satisfy the small absorption rate condition. The above parameter values result in a phase rotation of about 0.01 . We assume $\bar{n}=10^{4}$ and $\theta=0.01$ as typical values throughout this report. The error probability is estimated to be $P_{\mathrm{coh}}^{(\mathrm{min})}=0.14$ from Eqs. (11) and (15). The estimated error probability fails to satisfy the required values of $10^{-2}-10^{-4}$ for quantum computing $[15,16]$.

We will now show the improvement of the error probability by using the squeezed coherent state $|\xi, \alpha\rangle$. The squeeze operator for a single mode is [17]

$$
\hat{S}(\xi) \equiv e^{\frac{\xi}{2}\left[\hat{a}^{2}-\left(\hat{a}^{\dagger}\right)^{2}\right]},
$$

where $\xi=r e^{i \varphi}$, described with the amplitude $r$ and phase $\varphi$ (the direction of the squeeze in the phase space) of the squeezing parameter. The squeezed coherent state can be obtained by $\hat{S}(\xi)|\alpha\rangle=|\xi, \alpha\rangle$ [17] (for $\alpha=0, \hat{S}(\xi)|0\rangle=|\xi\rangle$ is the squeezed vacuum). The squeezed coherent state can be experimentally generated by using nonlinear optical effects between the pump beam and signal beam [18]. When the signal beam is the coherent state, the (output) idler beam becomes the squeezed coherent state. When the signal beam is the vacuum state, the idler beam becomes the squeezed vacuum; therefore, the squeezed coherent state can also be obtained from the
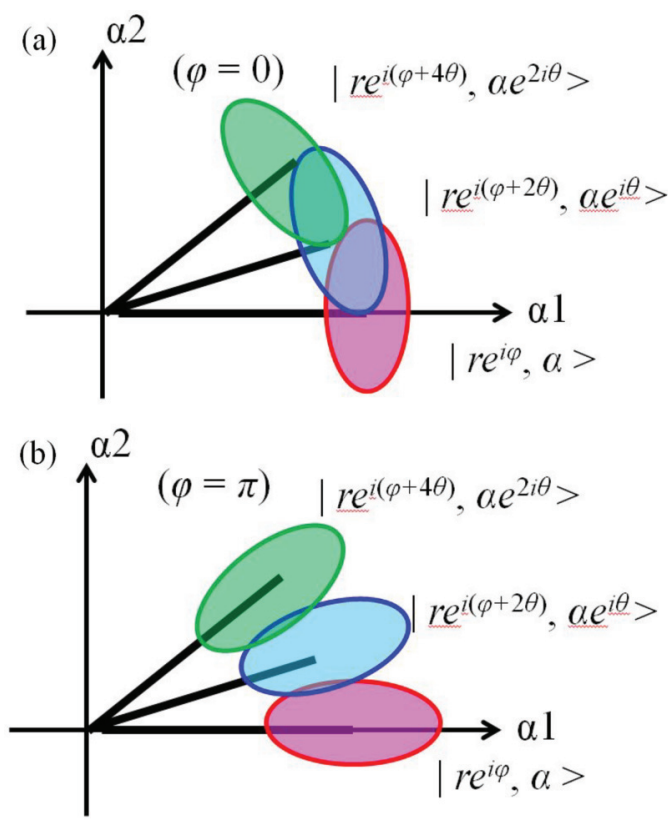

FIG. 3. (Color online) The states in phase space of the squeezed coherent states after interacting with two atoms. (a) The phase of the squeezing parameter, $\varphi=0$. The overlap between the three states $|\xi, \alpha\rangle,\left|\xi e^{i 2 \theta}, \alpha e^{i \theta}\right\rangle$, and $\left|\xi e^{i 4 \theta}, \alpha e^{i 2 \theta}\right\rangle$ is increased for the small value of the amplitude of the squeezing parameter $r$ and the small phase rotation angle $\theta$. (b) The phase of the squeezing parameter, $\varphi=\pi$. In this case, the overlap is decreased for all values of the amplitude of the squeezing parameter and the phase rotation angle.
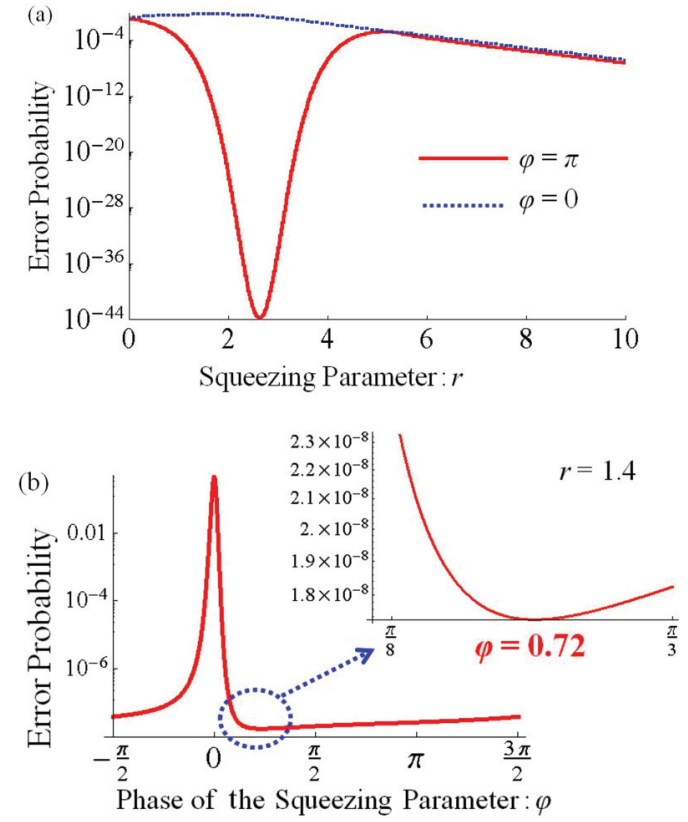

FIG. 4. (Color online) The error probability as a function of (a) the amplitude $r$ and (b) the phase $\varphi$ of the squeezing parameter for a fixed mean photon number $\bar{n}=10^{4}$ and $r=1.4$. The error probability is minimized at $r \sim 3$ for $\varphi=\pi$. The optimal value of the phase of the squeezing parameter $\varphi$ depends on $r$ when the mean photon number is fixed.

squeezed vacuum by the displacement operation. The overlaps of the photon states are reduced by using properly squeezed coherent states, as shown in Fig. 3. The power of discrimination of the squeezed coherent state between $|\xi, \alpha\rangle$ and $\left|\xi e^{i 2 \theta}, \alpha e^{i \theta}\right\rangle$ is estimated from the fidelity given by Peng and Li [17]:

$$
\begin{aligned}
F_{\mathrm{squ}}^{\prime}= & \left|\left\langle\xi, \alpha \mid \xi e^{i 2 \theta}, \alpha e^{i \theta}\right\rangle\right| \\
= & \frac{1}{\sqrt{\cosh ^{2} r-e^{i 2 \theta} \sinh ^{2} r}} \exp \\
& \times\left[\alpha^{2}\left(-1+\frac{e^{i \theta}+\left(1-e^{i 2 \theta}\right) \cos \varphi \cosh r \sinh r}{\cosh ^{2} r-e^{i 2 \theta} \sinh ^{2} r}\right)\right] .
\end{aligned}
$$

The fidelity $\left|\left\langle\xi e^{i 4 \theta}, \alpha e^{i 2 \theta} \mid \xi e^{i 2 \theta}, \alpha e^{i \theta}\right\rangle\right|$ is also written with the same formula as Eq. (17). Figure 4 plots the error probability as a function of the (a) amplitude $r$ and (b) phase $\varphi$ using Eqs. (11) and (17). Here, we fix the mean photon number at $\bar{n}=$ $\left|\alpha^{2}\right|\left[e^{-2 r} \cos \left(\theta-\frac{\varphi}{2}\right)+e^{2 r} \sin \left(\theta-\frac{\varphi}{2}\right)\right]+\sinh ^{2}(r)=10^{4}$ for a fair comparison by adjusting the amplitude to be $\alpha^{\prime}$ from the original value $\alpha$ as

$$
\alpha^{\prime}=\left|\sqrt{\frac{|\alpha|^{2}-\sinh ^{2}(r)}{e^{-2 r} \cos \left(\theta-\frac{\varphi}{2}\right)+e^{2 r} \sin \left(\theta-\frac{\varphi}{2}\right)}}\right|,
$$

since the mean photon number of the squeezed coherent state with amplitude $\alpha$ differs from that of the original coherent state with the same amplitude [8].

Figure 4 shows the error probability as a function of the amplitude of the squeezing parameter $r$. For $\varphi=0$ (the dotted line), the error probability is not reduced for $0 \leqslant r \leqslant 3$. For $\varphi=\pi$ (the solid line), the error probability is sharply reduced 

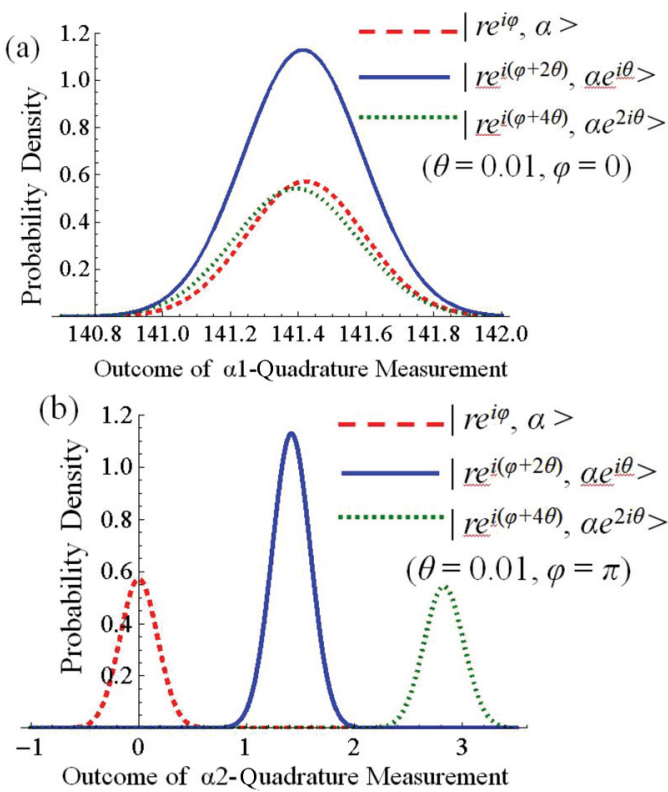

FIG. 5. (Color online) Probability density distributions for the outcomes of the quadrature measurement of the probe beam (a) corresponding to the quantum states in Fig. 4(a) projected on the $\alpha 1$ axis and (b) corresponding to the quantum states in Fig. 4(b) projected on the $\alpha 2$ axis.

for $0 \leqslant r \leqslant 3$ and the minimum value $P_{E}=1.42 \times 10^{-44}$ appears at $r=2.65$. The error probability increases for large squeezing because the amplitude $\alpha^{\prime}$ decreases to fix the mean photon number. The phase of the squeezing parameter $\varphi$ has to be optimized for each $r$, since the minimum conditions of the exponent in Eq. (17) are varied by using $\alpha^{\prime}$ given in Eq. (18). When we fix the amplitude $\alpha$ instead of the mean photon number, the optimal value of the phase is $\varphi=\pi$ and is independent of $r$. The optimal value of the phase $\varphi$ and the experimentally reported maximum value of the amplitude of the squeezing parameter $r=1.4[19,20]$ yield the error probability $P_{\text {squ }}^{(\min )}=1.73 \times 10^{-8}$.

\section{ERROR PROBABILITY IN HOMODYNE MEASUREMENT}

We will calculate the error probability for homodyne measurements, as the minimum error discrimination may be difficult to implement for coherent states and squeezed coherent states. In a homodyne measurement, the quantum state is projected onto a projection axis. Since the light to be measured is in the superposition state, as shown in Fig. 3, the probability density distributions shown in Fig. 5 can be obtained by projective measurement [in Fig. 5(a), projected onto the $\alpha 1$ axis; in Fig. 5(b), projected onto the $\alpha 2$ axis]. The error in entanglement generation in the homodyne measurement occurs due to overlaps of the probability density distributions representing the measurement outcome.

There is a formula for the probability density distributions when squeezed coherent states are projected onto the $x_{\lambda}$ axis (where $\lambda$ is the projective angle) [21]. We can expand the formula to include the phase shift $\theta$ as

$$
P_{\alpha, \xi}\left(x_{\lambda}, \theta\right)=\left(2 \pi \Delta x_{\lambda}^{2}\right)^{-1 / 2} \exp \left\{-\frac{\left(x_{\lambda}-\left\langle\hat{x}_{\lambda}\right\rangle\right)^{2}}{2 \Delta x_{\lambda}^{2}}\right\},
$$

where the expectation value of a quadrature operator

$$
x_{\lambda}=\frac{1}{\sqrt{2}}\left[\hat{a} \exp (-i \lambda)+\hat{a}^{\dagger} \exp (i \lambda)\right]
$$

is

$$
\begin{aligned}
\left\langle\hat{x}_{\lambda}\right\rangle & =\left\langle\alpha, \xi\left|\hat{x}_{\lambda}\right| \alpha, \xi\right\rangle \\
& =\frac{1}{\sqrt{2}}\left[\alpha \exp (-i \lambda)+\alpha^{*} \exp (i \lambda)\right] \exp (i \theta),
\end{aligned}
$$

and the expectation value of $\hat{x}_{\lambda}^{2}$ is

$$
\begin{aligned}
\left\langle\hat{x}_{\lambda}^{2}\right\rangle= & \left\langle\alpha, \xi\left|\hat{x}_{\lambda}^{2}\right| \alpha, \xi\right\rangle \\
= & \frac{1}{2}\left[\exp (2 r) \sin ^{2}\left(\lambda-\frac{\varphi}{2}+\theta\right)\right. \\
& \left.+\exp (-2 r) \cos ^{2}\left(\lambda-\frac{\varphi}{2}+\theta\right)\right] \\
& +\frac{1}{\sqrt{2}}\left[\alpha \exp (-i \lambda)+\alpha^{*} \exp (i \lambda)\right] \exp (i \theta) .
\end{aligned}
$$

Therefore, the variance of $\hat{x}_{\lambda}$ is given by

$$
\begin{aligned}
\Delta x_{\lambda}^{2}= & \left\langle\hat{x}_{\lambda}^{2}\right\rangle-\left\langle\hat{x}_{\lambda}\right\rangle^{2} \\
= & \frac{1}{2}\left[\exp (2 r) \sin ^{2}\left(\lambda-\frac{\varphi}{2}+\theta\right)\right. \\
& \left.+\exp (-2 r) \cos ^{2}\left(\lambda-\frac{\varphi}{2}+\theta\right)\right] .
\end{aligned}
$$

When the projection angle $\lambda=0$, the quantum states projected in the direction of the $\alpha 1$ axis yield probability density distributions as shown in Fig. 5(a). On the other hand, when $\lambda=\pi / 2$, the quantum states projected in the direction of the $\alpha 2$ axis yield the distributions shown in Fig. 5(b).

As mentioned above, since the error occurs from the overlaps between these probability density distributions, we will calculate these overlaps. The error probability is obtained with a rigorous method by subtracting the overlap between the probability density distributions of $|\xi, \alpha\rangle$ and $\left|\xi e^{i 4 \theta}, \alpha e^{i 2 \theta}\right\rangle$ from the sum of the overlaps between $|\xi, \alpha\rangle$ and $\left|\xi e^{i 2 \theta}, \alpha e^{i \theta}\right\rangle$ and the overlaps between $\left|\xi e^{i 2 \theta}, \alpha e^{i \theta}\right\rangle$ and $\left|\xi e^{i 4 \theta}, \alpha e^{i 2 \theta}\right\rangle$. The overlap between the probability density distributions of two quantum states of light (with phases $\theta_{1}$ and $\theta_{2}$ ) projected on a projection axis $x_{\lambda}$ can be described as

$$
P_{\text {squ }}^{\prime(\text { hom })}\left(\alpha, \xi, \theta_{1}, \theta_{2}\right)= \begin{cases}c_{A} \int_{\alpha^{\prime \prime} \eta\left(\theta_{1}, \theta_{2}\right)}^{\infty} P_{\alpha, \xi}\left(x_{\lambda}, \theta_{1}\right) d x_{\lambda}+c_{B} \int_{-\infty}^{\alpha^{\prime \prime} \eta\left(\theta_{1}, \theta_{2}\right)} P_{\alpha, \xi}\left(x_{\lambda}, \theta_{2}\right) d x_{\lambda} & \left(\frac{\theta_{2}-\theta_{1}}{2} \leqslant \lambda \leqslant \pi+\frac{\theta_{2}-\theta_{1}}{2}\right), \\ c_{B} \int_{\alpha^{\prime \prime} \eta\left(\theta_{1}, \theta_{2}\right)}^{\infty} P_{\alpha, \xi}\left(x_{\lambda}, \theta_{2}\right) d x_{\lambda}+c_{A} \int_{-\infty}^{\alpha^{\prime \prime} \eta\left(\theta_{1}, \theta_{2}\right)} P_{\alpha, \xi}\left(x_{\lambda}, \theta_{1}\right) d x_{\lambda} & \text { (otherwise) }\end{cases}
$$


where

$$
\alpha^{\prime \prime}=\mid \sqrt{|\alpha|^{2}-\sinh ^{2}(r) \mid}
$$

and

$$
\eta\left(\theta_{1}, \theta_{2}\right)=\frac{1}{2 \sqrt{2}}\left[\cos \lambda\left(\cos \theta_{1}+\cos \theta_{2}\right)+\sin \lambda\left(\sin \theta_{1}+\sin \theta_{2}\right)\right] .
$$

Factors $c_{A}$ and $c_{B}$ are the squares of the probability amplitudes (i.e., probabilities) in Eq. (8). That is, $c_{A}=1 / 4$ and $c_{B}=1 / 2$ for $P_{\mathrm{squ}}^{\prime(\mathrm{hom})}(\alpha, \xi, 0, \theta)$, and $c_{A}=1 / 2$ and $c_{B}=$ $1 / 4$ for $P_{\text {squ }}^{\prime(\text { hom })}(\alpha, \xi, \theta, 2 \theta)$. Furthermore, when these quantum states are symmetric to the transformation $\left|\xi e^{i 2 x}, \alpha e^{i x}\right\rangle \rightarrow$ $\left|\xi e^{i 2(2 \theta-x)}, \alpha e^{i(2 \theta-x)}\right\rangle$, the overlaps $P_{\text {squ }}^{\prime(\text { hom }}(\alpha, \xi, 0, \theta)$ and
$P_{\text {squ }}^{\prime(\text { hom })}(\alpha, \xi, \theta, 2 \theta)$ are the same. Here, $\alpha^{\prime \prime} \eta\left(\theta_{1}, \theta_{2}\right)$ represents the distance from the origin to the midpoint (the crossover point) between the two arbitrary probability density distributions $P_{\alpha, \xi}\left(x_{\lambda}, \theta_{1}\right)$ and $P_{\alpha, \xi}\left(x_{\lambda}, \theta_{2}\right)$. Equation (25) represents the condition for the fixed mean photon number as in Eq. (18). The reason why Eq. (25) differs from Eq. (18) is that the squeezed state $|\alpha, \xi\rangle$ differs from the $|\xi, \alpha\rangle$ used in the probability density distribution in Eq. (19). However, from the relation $|\alpha, \xi\rangle=\hat{D}(\alpha) \hat{S}(\xi)|0\rangle=\hat{S}(\xi) \hat{D}\left(\alpha \cosh r+\alpha^{*} e^{i \varphi} \sinh r\right)|0\rangle$, as $\alpha \cosh r+\alpha^{*} e^{i \varphi} \sinh r=\beta,|\alpha, \xi\rangle$ and $|\xi, \beta\rangle$ are essentially equivalent.

We can estimate the error probability of postselecting the rotated squeezed coherent state $\left|\xi e^{i 2 \theta}, \alpha e^{i \theta}\right\rangle$ from three states $|\xi, \alpha\rangle,\left|\xi e^{i 2 \theta}, \alpha e^{i \theta}\right\rangle$, and $\left|\xi e^{i 4 \theta}, \alpha e^{i 2 \theta}\right\rangle$. The error probability becomes

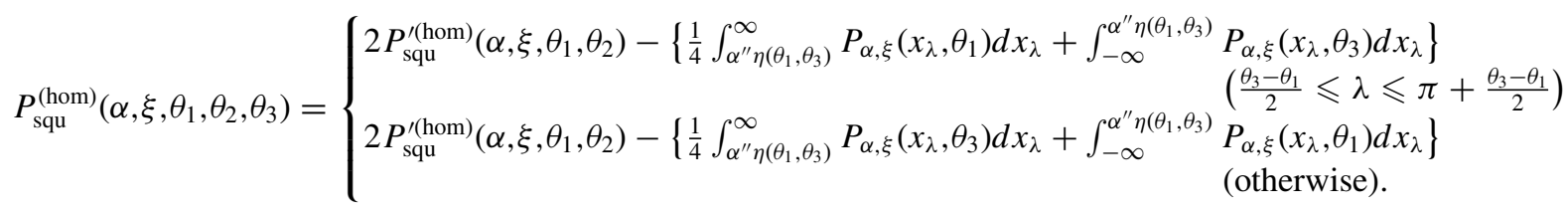

Figure 6 plots the error probability as a function of the projective angle $\lambda$. In the homodyne measurement, unlike in the optimal measurement, the optimal phase of the squeezing parameter $\varphi$ is $\varphi=\pi$. The squeezed states of the amplitude of the squeezing parameter $r=1.4$ yield the error probability $P_{\mathrm{squ}}^{(\text {hom })}=4.09 \times 10^{-5}$ under the following conditions: the optimal projective angle $\lambda=\left[\pi+\left(\theta_{3}-\theta_{1}\right)\right] / 2$, the fixed mean photon number $\bar{n}=10^{4}$, and the phase rotation angles $\theta_{1}=0, \theta_{2}=0.01$, and $\theta_{3}=0.02$. The error probability is about a thousand times as large as that obtained from the optimal measurement. Nevertheless, it is still small enough to satisfy the requirement for quantum computing.

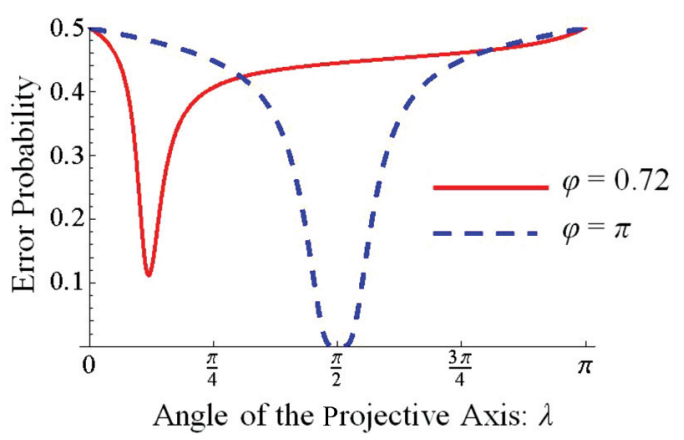

FIG. 6. (Color online) The error probability in the homodyne measurement as a function of the angle of the projective axis $\lambda$ under a fixed photon number. The solid line corresponds to the optimal phase of the squeezing parameter $\varphi=0.72$ in the optimal measurement obtained in Sec. III, and the dashed line corresponds to $\varphi=\pi$. In the homodyne measurement, the optimal phase of the squeezing parameter $\varphi$ is $\varphi=\pi$.

\section{DISCUSSION AND CONCLUSION}

We proposed entanglement generation between two atoms by communication using squeezed light. The squeezed light reduced the error probability by increasing the distance between quantum states of light in phase space. For typical values of $\alpha=100$ and $\theta=0.01$, the error probability, which is estimated to be $P_{\mathrm{coh}}^{(\mathrm{min})}=0.14$ for coherent light, can be reduced to $P_{\mathrm{squ}}^{(\mathrm{min})}=1.73 \times 10^{-8}$ with the amplitude of the squeezing parameter $r=1.4$ and the optimal phase of the squeezing parameter $\varphi$. In addition, we estimated the error probability of entanglement generation with the homodyne measurement to be $P_{\text {squ }}^{(\text {hom })}=4.09 \times 10^{-5}$ (for coherent states, $P_{\text {coh }}^{\text {(hom }}=0.23$ ).

In a quantum bus (a qubus), the success probability for entanglement generation is $1 / 2$. Such quantum gates can be qualified as probabilistic two-qubit quantum gates (PTQGs). As analyzed in the previous sections, the error occurs due to overlaps between quantum states of light. Since the squeezed coherent states and the coherent states of light are nonorthogonal, complete discernment cannot be performed. Here, we considered the possibility of error correction in the PTQG for error probabilities $P_{\text {squ }}^{(\mathrm{min})}$ and $P_{\text {squ }}^{\text {(hom })}$. Knill's error-correcting $C_{4} / C_{6}$ architecture $[9,10]$ can be implemented for the PTQG but requires a high success probability $(\sim 0.9)$. This method can correct errors in PTQGs up to an error probability of $1 \%$, if the success probability of the gates approaches $100 \%$. Repeated operations are thus required for the PTQG in order to achieve a success probability close to $100 \%$. Even if the success probability is improved to $100 \%$, the error probabilities $P_{\text {coh }}^{(\min )}$ and $P_{\text {coh }}^{(\text {hom }}$ for the qubus entangler with coherent states of light are too large to implement Knill's error-correcting $C_{4} / C_{6}$ 
architecture with $\alpha=100$ and $\theta=0.01$. Squeezed coherent states reduce the error probabilities so that Knill's architecture can be applied. Fujii and Tokunaga developed a fault-tolerant scheme based on topological one-way computation (TOWC) [16], which works with the PTQG with a lower success probability compared with Knill's architecture. In particular, TOWC can correct errors in PTQGs with a success probability of $1 / 2$ up to an error probability of $4.0 \times 10^{-4}$. Therefore, the error can be corrected for the qubus PTQG with a squeezed coherent light of $r=0.96$ (8.34 dB squeezing) if the light states are measured by minimum error discrimination and with that of $r=1.25$ (10.86 dB squeezing) if homodyne measurement is applied. Since the scheme based on TOWC currently has the highest error resilience, it is worth studying the implementation of fault-tolerant TOWC by a qubus PTQG with squeezed coherent states.

We will now list issues for future study. First, the present work neglects the transmission loss in short connections in quantum computers. Nevertheless, coupling loss and measurement loss still occur in real systems. The effects of such losses can be examined using the beam splitter model [22], and losses for a qubus PTQG using coherent light have already been analyzed [6]. We will go on to investigate the effects of loss for the squeezed coherent state. Second, to implement a measurement-based quantum computer, it is necessary to consider the method of generating cluster states from the two-partite entanglement described in the present study. The analysis of cluster states generation using a qubus PTQG has also been studied with coherent states [23-26]. It would be straightforward to expand the analysis for a qubus PTQG with squeezed coherent states. Third, we assumed that squeezed coherent light would be available and have shown that a qubus PTQG using squeezed coherent light works with a low error probability. In principle, such squeezed coherent light can be obtained by displacing the vacuum squeezed state. However, displacement from a squeezed vacuum to a bright squeezed light (with a mean photon number of $\bar{n} \sim 10^{4}$ ) may be restricted experimentally. The realization of bright squeezed light is thus a research task for the future.
[1] M. A. Nielsen and I. L. Chuang, Quantum Computation and Quantum Information (Cambridge University Press, Cambridge, 2000) .

[2] C. Negrevergne, T. S. Mahesh, C. A. Ryan, M. Ditty, F. CyrRacine, W. Power, N. Boulant, T. Havel, D. G. Cory, and R. Laflamme, Phys. Rev. Lett. 96, 170501 (2006).

[3] R. Raussendorf and H. J. Briegel, Phys. Rev. Lett. 86, 5188 (2001).

[4] T. Spiller, K. Nemoto, S. L. Braunstein, W. J. Munro, P. van Loock, and G. J. Milburn, New J. Phys. 8, 30 (2006).

[5] T. D. Ladd, P. van Loock, K. Nemoto, W. J. Munro, and Y. Yamamoto, New J. Phys. 8, 184 (2006).

[6] S. G. R. Louis, W. J. Munro, T. P. Spiller, and K. Nemoto, Phys. Rev. A 78, 022326 (2008).

[7] F. Y. Hong and S.-J. Xiong, Eur. Phys. J. D 54, 131 (2009).

[8] L. Praxmeyer and P. van Loock, Phys. Rev. A 81, 060303(R) (2010).

[9] E. Knill, R. Laflamme, and G. J. Milburn, Nature (London) 409, 4 (2001).

[10] H. Goto and K. Ichimura, Phys. Rev. A 80, 040303(R) (2009).

[11] J. Martin, J. Lumin. 81, 237 (1999).

[12] F. Jelezko, T. Gaebel, I. Popa, A. Gruber, and J. Wrachtrup, Phys. Rev. Lett. 92, 076401 (2004).

[13] C. W. Helstrom, Quantum Detection and Estimation Theory (Academic, New York, 1976).

[14] S. J. Devitt, A. D. Greentree, R. Ionicioiu, J. L. O’Brien, W. J. Munro, and L. C. L. Hollenberg, Phys. Rev. A 76, 052312 (2007).
[15] P. Lambropoulos and D. Petrosyan, Fundamentals of Quantum Optics and Quantum Information (Springer, Berlin, 2006), Chap. 10.

[16] K. Fujii and Y. Tokunaga, Phys. Rev. Lett. 105, 250503 (2010).

[17] J.-S. Peng and G.-X. Li, Introduction to Modern Quantum Optics (Academic, New York, 1998).

[18] M. Fox, Quantum Optics: An Introduction (Oxford University Press, Oxford, 2006).

[19] M. Mehmet, S. Ast, T. Eberle, S. Steinlechner, H. Vahlbruch, and R. Schnabel, Opt. Express 19, 25763 (2011).

[20] T. Eberle, S. Steinlechner, J. Bauchrowitz, V. Händchen, H. Vahlbruch, M. Mehmet, H. Müller-Ebhardt, and R. Schnabel, Phys. Rev. Lett. 104, 251102 (2010).

[21] S. M. Barnett and P. M. Radmore, Methods in Theoretical Quantum Optics (Oxford University Press, Oxford, 1997), Chap. 3.

[22] U. Leonhardt, Measuring the Quantum State of Light (Cambridge University Press, Cambridge, 1997), Chap. 4.

[23] S. G. R. Louis, K. Nemoto, W. J. Munro, and T. P. Spiller, Phys. Rev. A 75, 042323 (2007).

[24] S. G. R. Louis, K. Nemoto, W. J. Munro, and T. P. Spiller, New J. Phys. 9, 193 (2007).

[25] C. Horsman, K. L. Brown, W. J. Munro, and V. M. Kendon, Phys. Rev. A 83, 042327 (2011).

[26] K. L. Brown, C. Horsman, V. Kendon, and W. J. Munro, Phys. Rev. A 85, 052305 (2012). 